\title{
Oocyte and embryo metabolomics
}

\author{
Rebecca L. Krisher \\ Research Director, National Foundation for Fertility Research, Lone Tree, CO USA
}

\begin{abstract}
There is still much we do not understand about the metabolic requirements of oocytes and embryos. Their remarkable metabolic plasticity during in vitro culture has hampered our ability to make significant advances in culture media design that would better support optimal physiology. A new tool, metabolomics, may revolutionize what we know about the interactions between embryos and their culture environment. Armed with this knowledge, we may design more effective culture systems as well as discover metabolic biomarkers that predict oocyte and embryo viability. The objectives of this review are to introduce oocyte and embryo metabolism, review the current state of knowledge in the field, discuss the possibility that oocyte and embryo metabolism is significantly more complex than we have previously realized, examine a metabolomics dataset, and discuss how metabolomics may play a role in furthering our understanding of this exciting field that so significantly impacts the success of in vitro embryo production.
\end{abstract}

\section{Introduction}

Although incremental improvements have been made in the culture of oocytes and embryos from domestic species in the last decade, significant progress in improving in vitro maturation (IVM), fertilization (IVF) and culture (IVC) technologies remains elusive. Optimizing embryo culture media has been difficult, in part because we do not fully understand what the embryo requires in vitro to support successful development. Surprisingly, identifying the nutrient requirements of the early embryo has been difficult due to the plasticity of the embryo, rather than an extreme sensitivity of the embryo to culture conditions. Although the proportion of embryos developing to the blastocyst stage is affected by the composition of the culture medium, in most species some embryos are capable of successful development in a wide variety of media that often bear little resemblance to the composition of fluids of the follicle, oviduct and uterus. However, the metabolic costs of adaptation to suboptimal culture conditions can compromise embryo viability, cryotolerance, maintenance of pregnancy, fetal growth, and offspring health. Therefore, it is essential that embryo culture conditions provide the appropriate mixture of nutrients to support normal embryo physiology and to minimize adaptive stress and the associated risks to establishing pregnancy (Lane \& Gardner 2007).

Metabolomics technology has tremendous potential to expand our knowledge of embryo metabolism. In contrast to other methodologies currently in use, metabolomics provides a broader, more complex view of oocyte and embryo metabolism by examining multiple metabolic pathways within the context of an optimized culture system, rather than examining a single pathway or metabolite under artificial conditions. Metabolism of radiolabeled substrates, as well as fluorescent measurements of metabolite consumption and/or production, has been widely used to date to study the basic cellular metabolic pathways in embryos from multiple species. However, these 
techniques only examine individual metabolic pathways in isolation, without regard for other metabolic events or interactions between pathways. Using these techniques, we have established a very basic scheme for what we believe oocytes and embryos prefer in culture, but the metabolic pathways operating in oocytes and embryo are likely far more complex. Metabolomics technology allows us to develop a novel way of thinking about embryo metabolism; as a complex interplay of multiple metabolic mechanisms, versus the current dogma of a largely carbohydrate-based metabolism via glycolysis and oxidative phosphorylation. A metabolomics approach can provide information about not only suspected pathways of importance, but also about unknown regulatory mechanisms and metabolic intermediates. Only by understanding the relationships between various biochemical processes, including metabolism, energy production, control of reduction/ oxidation (redox) reactions, and cellular growth, can we begin to develop the next generation of in vitro culture systems that will result in increased embryo viability. Finally, oocyte and embryo metabolomics research has the potential to develop a non-invasive biomarker for viability, such that we could predict which embryos are most likely to result in a pregnancy following transfer, further increasing reproductive success.

\section{Measuring and interpreting oocyte and embryo metabolism}

A number of factors influence oocyte and embryo metabolism, which make it difficult to measure metabolic activity and complicate the interpretation of such measurements. Perhaps the most important consideration is that oocyte and embryo metabolism is not only affected by the conditions in which the oocyte or embryo develops, but also the medium in which metabolism is assessed (Gardner \& Leese 1990, Lane \& Gardner 1998, Krisher et al. 1999, Gandhi et al. 2001). Because an oocyte or embryo must be placed into artificial conditions, ie, in vitro culture, it is difficult, if not impossible, to define the normal metabolic profile of an in vivo matured oocyte or in vivo developed embryo. It is not currently possible to make metabolic measurements in situ, so the best we can do is evaluate oocytes or embryos immediately after recovery from the follicle, oviduct, or uterus. However, we cannot determine how much of the measured metabolic activity is attributable to normal physiology, or adaptation from in vivo to in vitro conditions. These considerations are further complicated when the media used in metabolic assays are formulated to contain radiolabelled substrates in the absence of other substrates, or in the presence of non-physiological concentrations of other substrates, to facilitate detection of changing concentrations of the substrate of interest. Due to these constraints, the nutrient preferences or requirements of oocytes and embryos have been defined as those conditions that produce an oocyte or embryo with similar metabolic activity to an in vivo-derived oocyte or embryo evaluated immediately after collection. However, the accuracy of this method is largely dependent not only on the medium used for the analysis but also on the number of metabolic pathways assessed. For example, rates of glycolysis between in vivo and in vitro derived embryos may be similar, but the rates of amino acid or lipid metabolism could be dramatically different between these same embryos. Metabolic measurements of oocytes are further complicated by the dynamic metabolic interactions occurring between the oocyte and the cumulus cells during maturation. Oocyte metabolism is likely altered when cumulus cells are removed (Heller et al. 1981, Eppig 1991, Saito et al. 1994, Downs 1995, Downs et al. 2002). Even given these caveats, metabolic studies have provided important information that has helped us begin to understand metabolic mechanisms in oocytes and embryos.

The oocyte

In vitro maturation typically results in reduced oocyte competence, or quality. This is despite the fact that meiosis (nuclear maturation) typically occurs successfully. Other processes, 
collectively referred to as cytoplasmic maturation, must also take place for an oocyte to have full developmental competence (Eppig 1996, Eppig et al. 1996). One of the most important of these processes, and one that can predict quality, is oocyte metabolism. In pigs, transfer of IVM, IVF and IVC produced embryos has resulting in offspring (Yoshida et al. 1993, Machaty et al. 1998, Kikuchi et al. 1999). However, the majority of immature porcine oocytes placed into an IVM system do not develop to the blastocyst stage, suggesting inadequate oocyte maturation (Singh et al. 1997). We have demonstrated that in vivo and in vitro matured pig oocytes have significantly different metabolic profiles, with in vitro matured oocytes being less active metabolically particularly in regards to pentose phosphate pathway (PPP) activity (Krisher et al. 2007).

In general, oocytes metabolize glucose via glycolysis, the PPP and the tricarboxylic acid (TCA) cycle, as shown in mice (Downs \& Utecht 1999), cats (Spindler et al. 2000), cattle (Rieger \& Loskutoff 1994, Krisher \& Bavister 1999), sheep (O'Brien et al. 1996) and pigs (Krisher et al. 2007). Elevated glycolytic and PPP activity is associated with the resumption and progression of meiosis (Tsutsumi et al. 1992, Zuelke \& Brackett 1992, Rieger \& Loskutoff 1994, Downs et al. 1996, Downs \& Utecht 1999, Steeves \& Gardner 1999, Cetica et al. 2002). Glycolysis (Fig. 1 blue arrows) contributes to ATP production as well as playing a role in maintaining redox potential in the oocyte, so it is not unexpected that glucose metabolism via this pathway plays a role in oocyte metabolism and competence. The PPP, while not directly involved in energy production, also appears to play a critical role in the oocyte by providing reducing power (NADPH), ribose sugars for DNA and RNA synthesis, and products used for purine synthesis. Pyruvate, derived from glucose or the maturation medium, and amino acids may be metabolized through the TCA cycle to produce ATP (Fig. 1 green and pink arrows). Amino acids are utilized by the cumulus oocyte complex (COC), and improve developmental competence to the blastocyst stage when included in IVM media (Banwell \& Thompson 2008). ATP content is important to the oocyte to provide energy for developmental events (Quinn \& Wales 1973, Van Blerkom et al. 1995), but quantity does not appear to be directly related to oocyte competence in pigs (Brad et al. 2003, Herrick et al. 2003, Krisher et al. 2007).

There are some species differences in oocyte metabolism. The mouse oocyte appears to prefer pyruvate, and this substrate alone can support maturation even without cumulus cells (Biggers et al. 1967, Leese \& Barton 1984, Fagbohun \& Downs 1992, Downs \& Mastropolo 1994, Downs \& Hudson 2000). In general, in the mouse cumulus cells take up and metabolize glucose, and then deliver glucose, pyruvate and lactate to the oocyte via the gap junctions between the two cell types (Saito et al. 1994, Downs \& Utecht 1999). In the bovine oocyte, pyruvate metabolism also dominates (Rieger \& Loskutoff 1994, Sutton et al. 2003). However, there appears to be metabolic interactions between glucose and pyruvate that may affect our interpretation of published results, thus glucose may play a larger role in bovine oocyte metabolism than in the mouse (Krisher \& Bavister 1999, Steeves \& Gardner 1999). Lipase activity in bovine oocytes suggests that oxidation of endogenous fatty acids (Fig. 1 orange arrow) may play a role in bovine oocyte maturation (Cetica et al. 2002). Our research has shown that, different from other species, mature, denuded porcine oocytes metabolize large amounts of glucose (Krisher et al. 2007). Pig oocytes contain a large amount of intracellular lipids that may play a significant role in energy production (Kikuchi et al. 2002, Sturmey \& Leese 2003a).

Metabolism of glucose, regardless of its relative contribution, may be the critical factor in determining oocyte competence (Krisher \& Bavister 1999, Sutton-McDowall et al. 2004). Ultimately, glucose metabolism mediates nuclear maturation and redox balance via PPP, cumulus expansion and glycosylation via the hexosamine biosynthetic pathway (HBP), and energy production via glycolysis in the oocyte (Fig. 1) (Sutton-McDowall et al. 2010). The HBP 


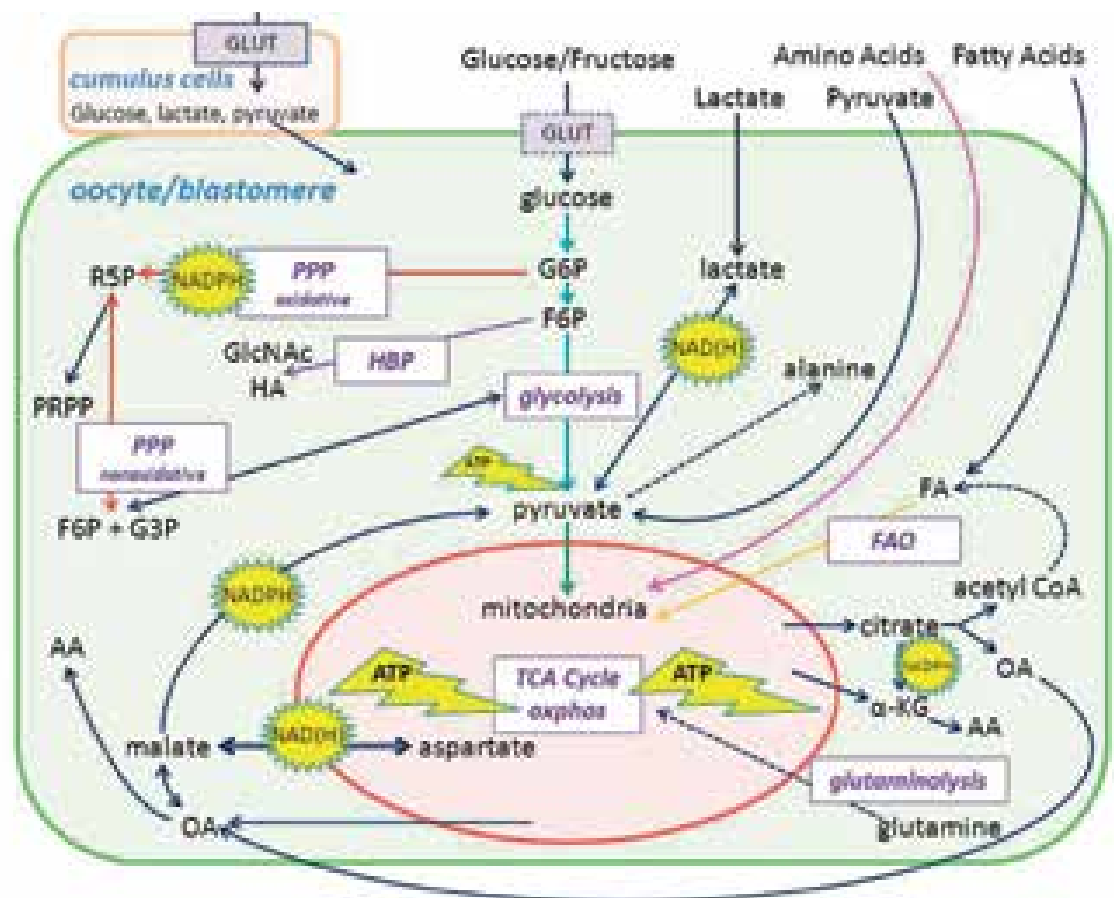

Fig. 1. An overview of the interrelated metabolic pathways that may function in mammalian oocytes and early embryos. Multiple energy substrates can be both taken up and produced by the oocyte or embryo to meet metabolic needs via multiple pathways, maintain redox and osmotic balance, and modify the external culture environment. The oocyte receives significant metabolic substrates via gap junction communication with cumulus cells (depicted in the upper left corner), while the embryo may take up a variety of substrates directly from the culture media. Dashed lines represent pathways that have only been hypothesized. Pathways names are shown in white boxes, and important end products in yellow. Glycolysis is highlighted by blue arrows, PPP by red arrows, hexosamine biosynthetic pathway by a purple arrow, and entry into the mitochondria for oxidative phosphorylation by a green arrow for pyruvate, a pink arrow for amino acids and an orange arrow for fatty acids. Abbreviations: $\alpha-K G$, alpha ketoglutarate; AA, amino acids; ATP; adenosine tri-phosphate; F6P, fructose 6 phosphate; FA, fatty acids; FAO, fatty acid $\beta$-oxidation; FAS, fatty acid synthase; G3P, glyceraldehyde 3 phosphate; G6P, glucose 6 phosphate; GlcNAc, N-Acetylglucosamine; GLUT, glucose transporters; HA, hyaluronic acid; HBP, hexosamine biosynthetic pathway; OA, oxaloacetate; Oxphos, oxidative phosphorylation; PRPP, phosphoribosyl pyrophosphate; R5P, ribose 5 phosphate; TCA, tricarboxylic acid.

(Fig. 1, purple arrows) metabolizes glucose to glucosamine, which is then used to produce extracellular matrix (hyaluronic acid) during cumulus cell expansion (Sutton-McDowall et al. 2006). The HBP also participates in the production of N-acetylglucosamine (GlcNAc) from glucose, glutamine and acetyl coA, which is involved in O-linked glycosylation to control protein function (Sutton-McDowall et al. 2010). PPP (Fig. 1 red arrows) seems to be particularly important for nuclear maturation (Downs et al. 1998, Herrick et al. 2006). In bovine oocytes, low oxygen concentration and increased glucose levels result in better oocyte quality by decreasing oxidative stress and increasing glycolytic activity (Hashimoto et al. 2000). Elevated glucose metabolism is correlated with improved oocyte competence in cats and cattle (Krisher \& Bavister 1999, Spindler et al. 2000). Stimulation of the PPP in bovine COC increases blastocyst development, suggesting that this glucose metabolic pathway is critical for oocyte competence (Krisher 1999). Overall, active glucose metabolism appears to be a biomarker of competent 
oocytes. Certainly in pigs, in vivo matured oocytes that are more developmentally competent have more active glucose metabolism (Krisher et al. 2007). The role of glucose metabolism in redox control and macromolecular synthesis may be at the root of this association.

The embryo

In vitro production of porcine embryos, although successful, results in embryos with suboptimal developmental ability. Although a wide variety of culture conditions are utilized, blastocyst development is surprisingly consistent. In general, embryos must produce ATP to provide energy for the cell to create building blocks for growth and development and maintain homeostasis in response to environmental stress (e.g., changing osmolarity, $\mathrm{pH}$, oxidative stress). These basic biochemical processes interact, leading to a complex physiological interplay that impacts embryo quality and viability. Typically in cellular metabolism, glucose is metabolized through glycolysis to pyruvate, which then either enters TCA cycle and is oxidized to produce ATP, or is converted to lactate (Fig. 1, blue and green arrows). In this context, carbohydrate metabolism has been extensively studied in embryos. There have also been some reports describing the contribution of other metabolic substrates to embryo development and viability, including amino acids and more recently fatty acids (Fig. 1 pink and orange arrows). In reality, all of these substrates, along with others, likely play a role in embryonic development and viability.

The study of embryo metabolism often focuses on the fate of glucose and the carbohydrates derived from it. Lactate and pyruvate are typically thought of as substrates of the early cleavage stages, while glucose metabolism via glycolysis increases at the time of blastocyst formation, when higher glucose uptake is a signature of viability (Gardner \& Leese 1987, Gardner et al. 2001). Domestic species use more glucose in the cleavage stages than mouse embryos, although use still increases during blastocyst formation. In the pig, glucose metabolism via glycolysis is active at the cleavage stages, particularly in in vivo derived embryos, although glycolytic activity is less than that of blastocysts (Flood \& Wiebold 1988, Swain et al. 2002). Glucose metabolism via glycolysis of in vitro produced pre-compaction bovine and ovine embryos is similar to that in the pig, although blastocyst glycolytic activity is higher in these ruminant species (Thompson et al. 1991, Rieger et al. 1992, Gardner et al. 1993, Swain et al. 2002). Pyruvate metabolism, however, is much lower in pig preimplantation embryos than sheep, cattle or mouse embryos at a similar developmental stage, suggesting an increased reliance on glucose metabolism in pigs (Leese \& Barton 1984, Rieger et al. 1992, Gardner et al. 1993, Swain et al. 2002). Ruminant blastocysts have a limited ability to oxidize glucose or pyruvate via the TCA cycle compared to mice, while pigs are intermediate in this ability (Rieger et al. 1992, Gardner et al. 1993, Swain et al. 2002). Lactate production by the embryo has traditionally been considered an adaptation to suboptimal culture conditions due to inhibition of oxidative phosphorylation (Gardner \& Leese 1990), or as a means to generate NAD + to be used in glycolysis (Lane \& Gardner 2005) or redox control. In the last decade, a prevailing view has been that of the 'quiet embryo' (Leese 2002, Baumann et al. 2007, Leese et al. 2007, Leese et al. 2008), which states that viable embryos, because they do not need to repair damage caused by stress, have lower oxidative phosphorylation activity and consume less $\mathrm{O}_{2}$.

Glucose is present in the bovine oviduct at a concentration of $\sim 2.5 \mathrm{mM}$ (Hugentobler et al. 2008, Hugentobler et al. 2010). However, there are culture media that successfully support development of bovine preimplantation embryos with glucose (SOF; Tervit et al., 1972; Steves and Gardner, 1999b, Gandhi et al., 2000) and without glucose (CR1aa, (Rosenkrans \& First 1994); mSOF, (Takahashi \& First 1992)). Similar to the situation in the cow, the pig oviduct contains glucose $(0.25 \mathrm{mM}$ in the post ovulatory mated ampulla, (Nichol et al. 1992)), and there are successful pig embryo culture media that do (NCSU23, (Petters et al. 1990, Petters 
\& Wells 1993)) and do not (PZM; Yoshioka et al., 2002) contain glucose. A recent report demonstrates that the presence of glucose in porcine zygote medium (PZM) during the final stages of culture increases blastocyst survival and ATP content, and in combination with glycine increases blastocyst hatching (Mito et al. 2012). Because the cow and pig embryo are able to develop in vitro from the 1-cell stage to blastocyst without exogenous glucose, without any known detrimental consequences, it may be possible that in glucose free media pyruvate is converted to phosphoenolpyruvate (PEP) by mitochondrial enzymes, an energy consuming process. Then, PEP may participate in the reversible reactions of glycolysis to the point that intermediates are produced, which then enter the PPP. Alternatively, pig and cattle embryos may depend primarily on fatty acids and amino acids for energy production, with little reliance on carbohydrates.

\section{Fatty acid metabolism}

It is only recently that the role of fatty acid $\beta$ oxidation (FAO; Fig. 1 orange arrow) in oocytes and embryos has received much research attention. Recent evidence suggests that lipid metabolism may play a role in energy production in the oocyte and embryo (Sturmey et al. 2006, Sturmey et al. 2009b). In pig, cow and mouse oocytes, inhibition of FAO reduces oocyte competence (Sturmey \& Leese 2003b, Ferguson \& Leese 2006, Dunning et al. 2010), indicating FAO during oocyte maturation is essential for subsequent embryo development. Exogenous carnitine, an essential cofactor for $\mathrm{FAO}$, in oocyte maturation media increases the number of active mitochondria in porcine oocytes (Somfai et al. 2011b) and the percentage of oocytes attaining MII in mice (Downs et al. 2009, Dunning et al. 2011) and pigs (Somfai et al. 2011b). Interestingly, although glucose uptake did not change lactate production was decreased, suggesting a different fate for glucose when ATP is generated from lipid metabolism.

Triglyceride content decreases at the blastocyst and hatched blastocyst stage in in vivo and in vitro produced pig embryos, and cultured blastocysts have increased triglyceride content compared to higher quality blastocysts produced in vivo, suggesting that that lipid metabolism is related to quality in pigs (Romek et al. 2010, Romek et al. 2011). In pig embryos, although development was not reduced when FAO was inhibited, glucose metabolism was upregulated (Sturmey \& Leese 2008), again suggesting an adjustment of glucose metabolism, potentially towards oxidative phosphorylation to compensate for a loss of optimal ATP production via FAO. Cow embryos behave similarly when FAO is inhibited, although development is compromised (Ferguson \& Leese 2006). Mouse embryo development and cell number was decreased when FAO was blocked during culture (Hewitson et al. 1996). The addition of fatty acids or carnitine to stimulate FAO to oocyte and embryo culture medium has primarily shown positive effects on development, although results are variable due in part to differences in type and concentration of fatty acid used (Spindler et al. 2000, Leroy et al. 2005, Dunning et al. 2010, Marei et al. 2010, Somfai et al. 2011a, Van Hoeck et al. 2011, Wu et al. 2011). Interestingly, in pig embryos, blastomeres with higher lipid content are more likely to contribute to the inner cell mass than the trophectoderm of the resulting blastocyst (Kim et al. 2012), suggesting that lipid metabolism may play a critical role in cell viability and pluripotency.

\section{Amino acid turnover}

The fate of amino acids for ATP production or protein synthesis is not clear. Amino acid uptake and production by embryos has been examined using high performance liquid chromatography (HPLC) in oocytes and embryos of several species. Amino acid turnover has been correlated with 
DNA damage in porcine, bovine and human embryos (Sturmey et al. 2009a). In vitro produced bovine embryos depleted more amino acids from the media than those produced in vivo, and sex differences in amino acid metabolism were noted (Sturmey et al. 2010). In human embryos, amino acid turnover was significantly different between euploid and aneuploid embryos, and also differed with embryo sex (Picton et al. 2010). The consumption and production of glutamine and alanine, respectively, as well as utilization of a panel of amino acids including alanine, arginine, glutamine, leucine and tryptophan, were predictive of the ability of bovine oocytes to cleave and develop to the blastocyst stage (Hemmings et al. 2012). Turnover of several amino acids, including leucine, in human embryos appears to be predictive of developmental success (Houghton et al. 2002, Brison et al. 2004). Amino acid turnover is affected by oxygen concentration during culture and is correlated with developmental competence in mouse embryos (Wale \& Gardner 2012). During pig preimplantation development, amino acid uptake and depletion changes over time and between amino acids, and more closely reflects amino acid turnover in human embryos than that of the mouse or cow (Booth et al. 2005). A complex logistical regression model including turnover of 4 amino acids associated with blastocyst development was developed that could accurately predict blastocyst formation in pig embryos (Booth et al. 2007). One specific amino acid, arginine, whose transporter was identified using deep sequencing technology in embryos, improves development and quality of in vitro produced pig embryos (Li et al. 2007, Bauer et al. 2010). This suggests that amino acid metabolism is useful for prediction of developmental capacity (Houghton et al. 2002, Sturmey et al. 2008, Sturmey et al. 2010).

\section{Reduction-oxidation (redox) state}

Redox homeostasis refers to cellular processes that maintain the correct reduction-oxidation environment within the cell. The redox state of a cell is set by the ratios of the redox couples $\mathrm{NAD}+/ \mathrm{NADH}$ (which control the lactate:pyruvate ratio), NADP +/NADPH, and GSH/GSSG (reduced and oxidized glutathione, respectively). Redox signaling mediates the response of cells to oxidative stress via redox sensitive transcription factors such as hypoxia inducible factors (HIFs), and can result in alteration of gene expression and apoptosis. In the preimplantation embryo, a normal redox state is critically important for successful development (Harvey et al. 2002). In oocytes and embryos, the redox potential is reduced and maintained by the glutaredoxin and thioredoxin systems, both of which use NADPH to regenerate GSH (GonzalezFernandez et al. 2005, Dumollard et al. 2007, Yuan et al. 2012). During in vitro culture of porcine embryos, addition of reductive agents increases blastocyst development (Ozawa et al. 2006). In cattle embryos, an environment with low oxygen results in a more reduced redox state, increased HIF2 $\alpha$ expression and improved embryo viability (Harvey et al. 2004). These results demonstrate the importance of redox homeostasis in embryos. However, we are just beginning to understand the molecular mechanisms that participate in this delicate interplay, and we know very little about how these intracellular mechanisms relate to, or even predict, embryo quality.

\section{Metabolic networks in oocytes and embryos}

We have recently proposed a novel model to describe embryo metabolism in the context of a broad, interconnected network of metabolic mechanisms that influence embryo viability (Krisher \& Prather 2012). Although the TCA cycle is a much more efficient means of producing ATP when compared to glycolysis, the oocyte and embryo may have more important metabolic uses 
for glucose than simply ATP production. The oocyte must establish the foundation from which early embryo development can take place. This may include redox control and macromolecular synthesis, including DNA, RNA, proteins and lipids to support rapid embryonic growth. Thus, ATP may not be the primary driver of oocyte or embryo metabolism. All of these needs require glucose as a carbon source, consume TCA cycle intermediates, and require NADPH for reductive power. Thus, it may be too simplistic to view oocyte and embryo glucose metabolism as primarily a means to produce ATP, ignoring the embryo's biosynthetic requirements. In this case, fatty acids and/or amino acids likely support basal TCA activity to provide ATP.

There is some experimental evidence to support this hypothesis. In pig blastocysts, expression of genes involved in the Warburg Effect has been described (Redel et al. 2012). These genes may slow glucose metabolism through the TCA cycle and re-direct glucose metabolism towards the PPP and lactate production. Inhibition of TCA activity at the time of embryo compaction improves pig and cow embryo development (Thompson et al. 2000, Machaty et al. 2001, Rieger et al. 2002), potentially by reducing ATP production from glucose and forcing metabolism via the PPP to establish a favorable redox state within the embryo (Thompson et al. 2000). Stimulating PPP activity in 8-16 cell stage bovine embryos increases glucose metabolism without affecting lactate production, and reduces lipid accumulation (De La Torre-Sanchez et al. 2006).

\section{Metabolomics}

Metabolomics is the next iteration of 'omics' technologies. This comprehensive approach is a powerful tool to examine the metabolic result of changes in gene and protein expression during development and in response to environmental changes in a systems biology context (Fernie et al. 2004). It is only recently that technological advances in automation and information technology have allowed the basic techniques of metabolomics to be applied to these types of analyses, particularly to very small samples like oocytes and embryos. A variety of platforms may be utilized for metabolomic analysis, including gas or liquid chromatography (GC and LC, respectively) and/or matrix-assisted laser desorption/ionization (MALDI) coupled to mass spectrometry (MS), as well as nuclear magnetic resonance (NMR), Raman, or near infra-red (NIR) spectroscopy. If MS is used, measurement of the molecules' mass, or the masses of distinctive fragments of that molecule following derivitization, results in a specific molecular fingerprint that then allows identification of the metabolite when compared to known databases. Either targeted (measuring a predefined set of metabolites) or non-targeted (measuring all detectable metabolites, both known and unknown) approaches may be taken. While the non-targeted approach investigates a larger cohort of metabolites, the datasets suffer from excessive size and complexity. Luckily, informatics workflows for metabolomics have been developed that improve both metabolite annotation and interpretation, allowing for the simultaneous monitoring of thousands of metabolites. Metabolites may be individually identified by matching chemical signatures to metabolite databases, and unknown metabolites can be described by the signature itself. It is important to note that absolute quantitation is not necessary to make valid metabolomic comparisons. Although absolute quantitation is usually preferable, it is difficult to accomplish in large scale studies, time consuming, expensive, and can only be done for known compounds with readily available commercial standards. Often, both uniand multivariate statistical analyses enable recognition of differences or changes in metabolite profiles that can be used as markers of disease or toxicity, even before specific metabolites are identified. Metabolomics studies often examine urine, blood or plant extracts, samples that are not typically limiting in volume but that may have thousands of metabolites. In contrast, in metabolic studies of oocytes or embryos, samples are very limiting in both size and metabolite 
concentration but may contain only a few hundred metabolites. Several approaches may be taken, including analysis of spent media at various times during development, analysis of lysed oocytes or embryos in solution, or direct analysis of a single oocyte or embryo.

To date, metabolomics technology has only been minimally applied to examine the total metabolic activity of embryos during culture. In the field of human IVF, great interest exists in being able to predict and accurately choose the most viable embryo for transfer resulting in the best chance for a successful pregnancy. As in domestic animals, the most widely used parameter on which to base embryo transfer is currently embryo morphology, which has proven to be inaccurate. Metabolomic analysis of spent culture media has been proposed as a useful non-invasive biomarker for this purpose (Botros et al. 2008). Raman and NIR spectroscopy have been used to analyze spent culture media and compare the resulting metabolomic profiles of embryos that resulted in a pregnancy to those that did not. Although in the initial retrospective studies associations were observed between spent media profiles and the potential for successful implantation (Nagy et al. 2008, Sakkas et al. 2008, Scott et al. 2008, Marhuenda-Egea et al. 2011), randomized controlled trials did not result in any increase in pregnancy rate following selection using a metabolomic profile based viability score compared to morphology alone (Hardarson et al. 2012, Vergouw et al. 2012). Of interest, these studies did not identify specific metabolites, only calculated a viability index based upon the spectrum of unspent media. The goal here was biomarker-based prediction, not the generation of knowledge that would inform what we know of embryo metabolism. In similar work, electrospray ionization MS (ESI-MS) was used to differentiate between culture media from embryos that did and did not implant, although again few specific metabolites were identified (Cortezzi et al. 2013). Metabolomic profiling of embryo culture media using LC-MS has demonstrated that vitrification of human oocytes does not alter substrate metabolism of subsequently produced embryos (Dominguez et al. 2013).

Lipids are one class of metabolites that have begun to be studied in depth in oocytes and embryos using metabolomic techniques. Using MALDI-MS, the lipid content of individual oocytes and embryos from several species was defined, and alterations in lipid profile of bovine embryos due to culture with serum were described (Ferreira et al. 2010). MALDI time of flight (TOF) MS was used to evaluate the lipid profile of human cumulus cells, demonstrating that phosphatidylcholine might be used as a marker of oocytes capable of producing an embryo that results in pregnancy (Montani et al. 2012). Desorption electrospray ionization mass spectrometry (DESI-MS) has been used to describe changes in lipid profile during preimplantation development in the mouse, and described differences between embryos produced in vitro and in vivo (Ferreira et al. 2012). In bovine embryos, MALDI-MS revealed differences in phosphatidylcholine and sphingomyelin due to in vitro culture as well as subspecies of origin (Sudano et al. 2012).

We are working to optimize metabolomics methodology to assess both the composition of the media after culture, as well as the metabolite composition of the oocyte or embryo itself. When analyzing media following individual embryo culture in mouse and cattle, we have found that the combination of GC- and MALDI-MS results in a complete metabolite profile, including carbohydrates, amino acids and lipids. We are using MALDI-MS alone for analysis of both individual embryos and groups of embryos in solution. Our initial work suggests that a comprehensive analysis of embryo metabolism during development will shed new light on this area of research. In a preliminary experiment, we have described the uptake or production of metabolites from culture during blastulation of bovine and murine embryos (Fig. 2). Outbred (CF1) females were stimulated with PMSG/hCG and mated to B6D2F1 males to produce in vivo derived morulae. Bovine morulae were produced from abattoir derived oocytes using in vitro 

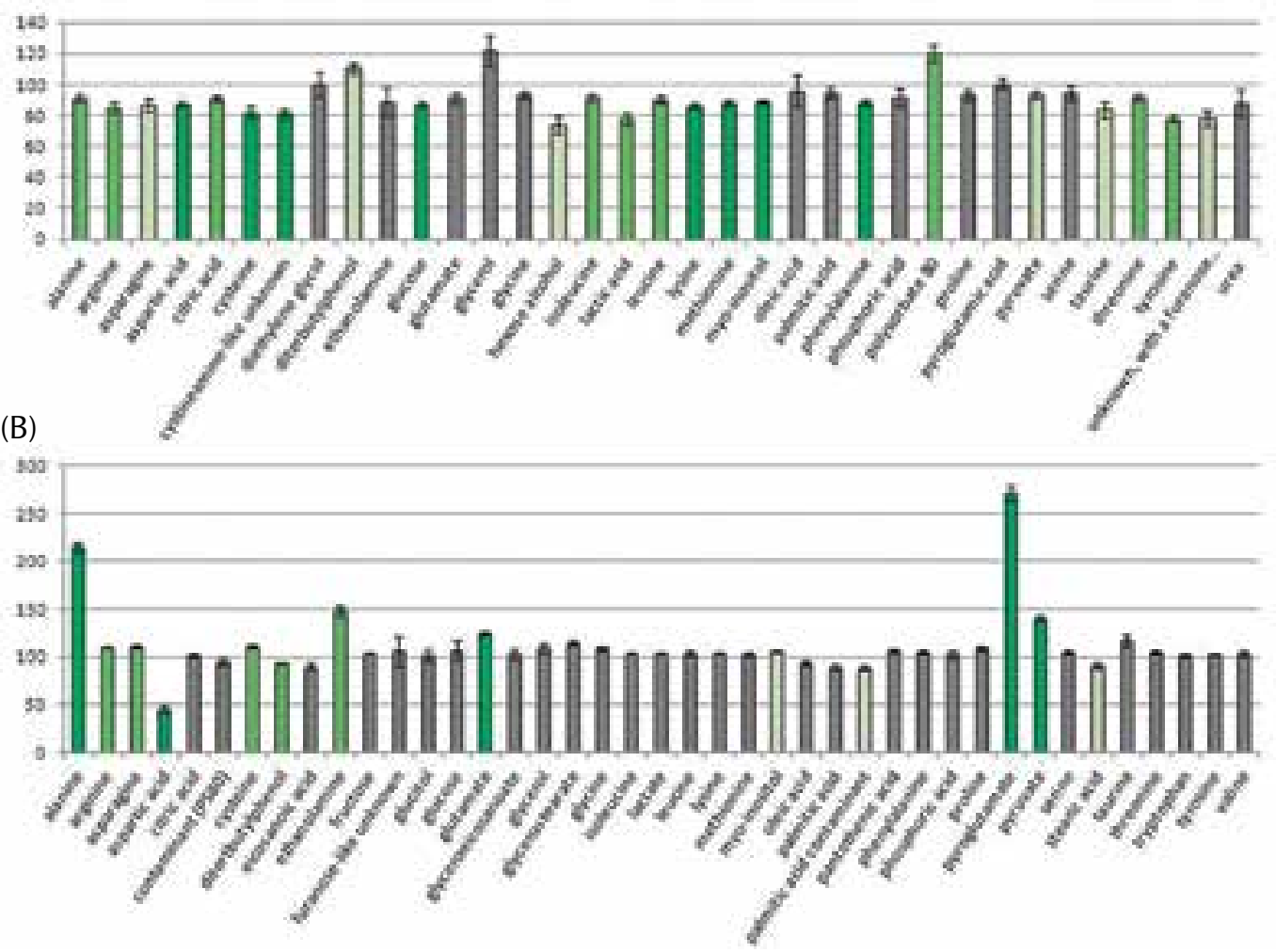

Fig. 2 A metabolomics dataset examining the in vitro metabolism of blastulating mouse and bovine embryos. A) Outbred mouse embryos, produced in vivo, were cultured for 24 hours (D3-D4) in vitro from the compact morula to the expanded blastocyst stage. B) In vitro produced bovine embryos were cultured for 48 hours (D5-D7) from the compact morula to hatching blastocyst stage. All media contained labeled palmitic acid. Media was subsequently examined by GC-MS. Media from drops with embryos was compared to control media without embryos for each metabolite. Dark green $p=<0.01$; medium green $\mathrm{p}<0.05$; light green $\mathrm{p}<0.09$, different than unspent media.

maturation, fertilization and culture. Morulae from both species were cultured individually in $7 \mu \mathrm{L}$ (murine) or $12 \mu \mathrm{L}$ (bovine) drops in media containing carnitine $(0.5 \mathrm{mM}$ murine, $1 \mathrm{mM}$ bovine), oleic acid, and $\mathrm{C}^{13}$ labeled palmitic acid (25 $\mu \mathrm{M}$ each fatty acid murine, $50 \mu \mathrm{M}$ each bovine). Culture media also contained $3 \mathrm{mM}$ glucose (murine) or fructose (bovine). After 24 hr (murine) or $48 \mathrm{hr}$ (bovine), development was assessed and media from drops with embryos (spent media, $\mathrm{n}=5-6 /$ species) that developed to the expanded (murine) or hatching (bovine) blastocyst stages was collected. Media from drops without embryos in the same dish was also collected (unspent media, $n=3-5 /$ species). An equal volume of an internal standard was added to each sample, and samples were derivatized with methoxyamine $\mathrm{HCl}$ followed by MSTFA $+1 \%$ TMCS. Samples were randomized and injected onto a GC-MS platform in duplicate. GC-MS spectra were screened in the Golm and NIST metabolite libraries to assign putative identifications to molecular features. Principal component analysis (PCA) was performed using SIMCA software. Raw peak areas were averaged and normalized to total ion signal intensity, and 1-way ANOVA used to test differences between spent and unspent media values for each metabolite. Using PCA analysis, we are able to see good separation of the spent and unspent media samples. These results demonstrate several interesting points, not the least of which is 
that a metabolomics approach can provide a comprehensive examination of multiple (up to 41) metabolites from a single embryo during in vitro culture.

First, mouse embryos appear to use a much wider variety of metabolites from the culture media than do cattle embryos (Fig. 2). We observed significant $(p<0.01)$ uptake of aspartic acid, cysteine, glucose, lysine, methionine, myo-inositol, and phenylalanine, as well as $(p<0.05)$ alanine, arginine, citric acid, isoleucine, lactic acid, leucine, threonine, and tyrosine from the culture medium by murine embryos. Following culture of bovine embryos, alanine, glutamate, pyroglutamate, and pyruvate $(p<0.01)$, as well as arginine, asparagine, and cysteine $(p<0.05)$ concentrations in the media were increased following embryo culture. Only aspartic acid was taken up $(p<0.01)$ in a significant amount by bovine embryos. Of interest fructose, the most abundant carbohydrate included in the bovine culture media, was not significantly consumed by blastocysts $(p>0.05)$. Neither oleic nor palmitic acids were significantly taken up by embryos of either species. Aspartic acid was the only metabolite significantly consumed from the culture media by both murine and bovine embryos, although alanine, arginine, and cysteine metabolism appear to play a dynamic role in embryos of both species. These data reveal that murine blastocysts use more exogenous metabolic substrates provided in the culture media than do bovine blastocysts, which may use endogenous energy stores such as lipids. However, the origin of the embryos examined (in vivo versus in vitro) may affect metabolic activity. Studies to compare in vivo and in vitro produced embryos in both species are underway to answer this question. Second, we detected multiple contaminants in the culture medium that we know are not intentionally added, since all of the media are produced in our laboratory. These contaminants include diethylene glycol, ditertbutylphenol, eicosanoic acid, ethanolamine, glucitol, glyceroeicosanoate, glycerostearate, glycerol, hexose alcohol, palmitic acid, a contaminant tentatively identified as polysorbate 80 , pyroglutamate, and stearic acid. We can distinguish between the palmitic acid that we add and the contaminant because labeled palmitic acid was used in the culture media. Some of these, such as ditertbutylphenol and diethylene glycol, may be derived from plasticware or the oil overlay. Others likely arise from the GC-MS instrument. We are currently identifying the source of as many of these unknowns as possible. Interestingly, some of them are significantly different in media before and after embryo culture. Additional experiments will determine whether these contaminants are actually utilized by embryos in culture, or are artifacts of the technology. Last, in contrast to published data that lactate is produced during in vitro embryo culture (Gardner \& Leese 1990, Lane \& Gardner 2005), in our experiment lactate was actually taken up by mouse embryos, and not used significantly by bovine embryos. This may be due to improvements in embryo culture media, or to the fact that by employing metabolomics we were able to examine the consumption or production of lactate in the context of a complete media rather than in isolation.

Metabolomic results such as these may have a significant impact on how embryo culture media is designed and how embryos are selected for transfer. Initially, it will be important that the metabolism of in vivo produced embryos be examined in this scenario, so that we understand the metabolic profile of high quality embryos and can try to achieve that in our in vitro produced embryos. However, these initial metabolic profiles do provide clues for embryo culture media improvement. For example, it seems that addition of palmitic or oleic acid, two of the most common fatty acids in reproductive fluids, to culture media is not useful for either murine or bovine embryos during the preimplantation period. The considerable production of alanine and pyroglutamate in bovine, but not murine, embryos raises many experimental questions. Alanine production has previously been noted in human and bovine embryo culture, and is thought to be a means of ridding the embryo of ammonium ions (Partridge \& Leese 1996, Houghton et al. 2002, Orsi \& Leese 2004). Alternatively, alanine production 
may reflect a need to keep excess pyruvate from entering the TCA cycle. Pyroglutamate (also known as 5-oxo-proline) can be used in N-terminal protein modification to control activity, as a reservoir of glutamate, and may play a role in osmoprotection (Kumar \& Bachhawat 2012). This may suggest that we should be providing the embryo with this substrate for correct protein function, or that we may be providing the embryo with an overabundance of glutamic acid. Alternatively, it is possible that the embryo must use glutamic acid to produce pyroglutamate to regulate osmolarity in a suboptimal in vitro environment, potentially robbing substrates and energy from other pathways to do so. Pyroglutamate is also a substrate of the $\gamma$-glutamyl cycle, which transports amino acids into cells and is involved in the turnover of glutathione. Thus, pyroglutamate may also be an indicator of oxidative stress. Significant research is warranted on this amino acid derivative that was previously not even considered in embryo culture. Another noticeable point is that aspartic acid is significantly taken up from the culture media by embryos of both species; bovine blastocysts take up over half of the available aspartic acid. In human embryos, aspartate is produced (Houghton et al. 2002), suggesting some interesting species differences that may be relevant when choosing an animal model for human embryo culture media development and research. Do bovine embryos require greater amounts of aspartic acid, even though they do not deplete the media? Aspartate is required in the malate-aspartate shuttle, which transports NADH into the mitochondria for oxidative phosphorylation. Aspartate can also be used for the production of other amino acids, including methionine, threonine, isoleucine, lysine and asparagine, all of which were significantly depleted from the culture medium by mouse blastocysts. Possibly bovine embryos primarily use aspartic acid in the production of these other amino acids, rather than taking them up from exogenous sources. There are some important issues to take into consideration when using metabolomic data for media development, however. Substrate functions other than metabolic should be considered prior to altering the media. Substrates may play an important role in osmotic regulation or antioxidant defense, for example. In addition, unless labeled substrates are used, this technology cannot differentiate between substrates from the culture media or those produced by the embryo itself. Thus, there may be significant uptake and production of a substrate that we do not observe simply because the net difference is zero.

Finally, it becomes possible to consider studying the complete metabolic profiles of embryos that successfully implant, as this can be accomplished non-invasively. Because blastocyst culture and vitrification is now commonly used in a variety of species, including human, time is afforded for examination of metabolic profiles of culture media to select the embryo most likely to implant prior to transfer. It seems likely, given the interdependent relationships between metabolic pathways, that a combination of metabolites, rather than just one substrate (ie glucose) or even one class of substrates (ie, amino acids) will most accurately predict implantation potential. Thus, we might think of a metabolomic profile examining the use and production of multiple substrates together as a biomarker of embryo competence.

This preliminary example demonstrates how metabolomics information is providing a completely new picture of preimplantation embryo metabolism, and will significantly change how we think about culturing embryos in vitro. We can begin to understand what metabolic pathways embryos prefer, and how they make metabolic compromises in suboptimal conditions that result in reduced viability. With this information, we can design novel culture media to best support normal physiology and thus long term developmental potential. Instead of providing a soup of random metabolites to the embryo in culture, some of which may be inhibitory or detrimental, we can provide the embryo with precisely what it needs to support optimal metabolism and thus viability. 


\section{Conclusion}

Oocyte and embryo metabolism is a critical factor in determining quality. However, to understand the relationship between metabolism and viability, we must examine the complex metabolic pathways in total and appreciate their interrelationships. Previous studies have provided only snapshots of metabolic pathways in isolation. However, application of metabolomic technologies to the analysis of oocyte and embryo metabolism permits visualization of metabolism in optimized culture conditions and in the context of the complete metabolic system. This information will likely revolutionize our understanding of oocyte and embryo metabolism. Thus informed, we can elucidate the complex metabolic relationships occurring and manipulate them in culture to produce and identify developmentally competent embryos.

\section{Declaration of interest}

The author declares that she has no actual or perceived conflict of interest that could be perceived as prejudicing the impartiality of the research reported.

\section{Acknowledgements}

The author would like to thank Dr. J.R. Herrick and Dr. A.L. Heuberger for critical review of the manuscript.

\section{References}

Banwell KM \& Thompson JG 2008 In vitro maturation of Mammalian oocytes: outcomes and consequences. Seminars in Reproductive Medicine 26 162-174.

Bauer BK, Isom SC, Spate LD, Whitworth KM, Spollen WG, Blake SM, Springer GK, Murphy CN \& Prather RS 2010 Transcriptional profiling by deep sequencing identifies differences in mRNA transcript abundance in in vivo-derived versus in vitro-cultured porcine blastocyst stage embryos. Biology of Reproduction 83 791-798.

Baumann CG, Morris DG, Sreenan JM \& Leese HJ 2007 The quiet embryo hypothesis: molecular characteristics favoring viability. Molecular Reproduction \& Development 74 1345-1353.

Biggers JD, D.G. W \& Donahue RP 1967 The pattern of energy metabolism in the mouse oocyte and zygote. The Proceedings of the National Academy of Sciences 58 560-567.

Booth PJ, Humpherson PG, Watson TJ \& Leese HJ 2005 Amino acid depletion and appearance during porcine preimplantation embryo development in vitro. Reproduction 130 655-668.

Booth PJ, Watson TJ \& Leese HJ 2007 Prediction of porcine blastocyst formation using morphological, kinetic, and amino acid depletion and appearance criteria determined during the early cleavage of in vitro-produced embryos. Biology of Reproduction 77 765-779.

Botros L, Sakkas D \& Seli E 2008 Metabolomics and its application for non-invasive embryo assessment in IVF. Molecular Human Reproduction 14 679-690.

Brad AM, Bormann CL, Swain JE, Durkin RE, Johnson
AE, Clifford AL \& Krisher RL 2003 Glutathione and adenosine triphosphate content of in vivo and in vitro matured porcine oocytes. Molecular Reproduction \& Development 64 492-498.

Brison DR, Houghton FD, Falconer D, Roberts SA, Hawkhead J, Humpherson PG, Lieberman BA \& Leese HJ 2004 Identification of viable embryos in IVF by non-invasive measurement of amino acid turnover. Human Reproduction 19 2319-2324.

Cetica P, Pintos L, Dalvit G \& Beconi M 2002 Activity of key enzymes involved in glucose and triglyceride catabolism during bovine oocyte maturation in vitro. Reproduction 124 675-681.

Cortezzi SS, Cabral EC, Trevisan MG, Ferreira CR, Setti AS, Braga DPAF, Figueira RCS, laconelli A, Jr, Eberlin MN, Borges E, Jr. 2013 Prediction of embryo implnatation potential by mass spectrometry fingerprinting of the culture medium. Reproduction published online before print February 12, 2013, doi: 10.1530/REP-12-0168.

De La Torre-Sanchez JF, Gardner DK, Preis K, Gibbons J \& Seidel GE, Jr. 2006 Metabolic regulation of in vitroproduced bovine embryos. II. Effects of phenazine ethosulfate, sodium azide and 2,4-dinitrophenol during post-compaction development on glucose metabolism and lipid accumulation. Reproduction, Fertility, \& Development 18 597-607.

Dominguez F, Castello D, Remohi J, Simon C, \& Cobo A 2013 Effect of vitrification on human oocytes: a metabolic profiling study. Fertility and Sterility 99 565-572.

Downs SM 1995 The influence of glucose, cumulus cells, 
and metabolic coupling on ATP levels and meiotic control in the isolated mouse oocyte. Developmental Biology 167 502-512.

Downs SM \& Hudson ED 2000 Energy substrates and the completion of spontaneous meiotic maturation. Zygote 8 339-351.

Downs SM, Humpherson PG \& Leese HJ 1998 Meiotic induction in cumulus cell-enclosed mouse oocytes: involvement of the pentose phosphate pathway. Biology of Reproduction 58 1084-1094.

Downs SM, Humpherson PG \& Leese HJ 2002 Pyruvate utilization by mouse oocytes is influenced by meiotic status and the cumulus oophorus. Molecular Reproduction and Development 62 113-123.

Downs SM, Humpherson PG, Martin KL \& Leese HJ 1996 Glucose utilization during gonadotropin-induced meiotic maturation in cumulus cell-enclosed mouse oocytes. Molecular Reproduction and Development 44 121-131.

Downs SM \& Mastropolo AM 1994 The participation of energy substrates in the control of meiotic maturation in murine oocytes. Developmental Biology 162 154-168.

Downs SM, Mosey JL \& Klinger J 2009 Fatty acid oxidation and meiotic resumption in mouse oocytes. Molecular Reproduction \& Development 76 844-853.

Downs SM \& Utecht AM 1999 Metabolism of radiolabeled glucose by mouse oocytes and oocyte-cumulus cell complexes. Biology of Reproduction 60 1446-1452.

Dumollard R, Duchen M \& Carroll J 2007 The role of mitochondrial function in the oocyte and embryo. Current Topics in Developmental Biology 77 21-49.

Dunning KR, Akison LK, Russell DL, Norman RJ \& Robker RL 2011 Increased beta-oxidation and improved oocyte developmental competence in response to I-carnitine during ovarian in vitro follicle development in mice. Biology of Reproduction 85 548-555.

Dunning KR, Cashman K, Russell DL, Thompson JG, Norman RJ \& Robker RL 2010 Beta-oxidation is essential for mouse oocyte developmental competence and early embryo development. Biology of Reproduction 83 909-918.

Eppig JJ 1991 Intercommunication between mammalian oocytes and companion somatic cells. Bioessays 13 569-574.

Eppig JJ 1996 Coordination of nuclear and cytoplasmic oocyte maturation in eutherian mammals. Reproduction, Fertility and Development 8 485-489.

Eppig JJ, O'Brien M \& Wigglesworth K 1996 Mammalian oocyte growth and development in vitro. Molecular Reproduction and Development 44 260-273.

Fagbohun CF \& Downs SM 1992 Requirement for glucose in ligand-stimulated meiotic maturation of cumulus cell-enclosed mouse oocytes. Journal of Reproduction and Fertility 96 681-697.

Ferguson EM \& Leese HJ 2006 A potential role for triglyceride as an energy source during bovine oocyte maturation and early embryo development. Molecular Reproduction and Development 73 1195-1201.

Fernie AR, Trethewey RN, Krotzky AJ \& Willmitzer L
2004 Metabolite profiling: from diagnostics to systems biology. Nature Reviews Molecular Cell Biology 5 763-769.

Ferreira CR, Pirro V, Eberlin LS, Hallett JE \& Cooks RG 2012 Developmental phases of individual mouse preimplantation embryos characterized by lipid signatures using desorption electrospray ionization mass spectrometry. Anal Bioanal Chem 404 29152926.

Ferreira CR, Saraiva SA, Catharino RR, Garcia JS, Gozzo FC, Sanvido GB, Santos LFA, Lo Turco EG, Pontes JHF, Basso AC, Bertolla RP, Sartori R, Guardieiro MM, Perecin F, Meirelles FV, Sangalli JR \& Eberlin MN 2010 Single embryo and oocyte lipid fingerprinting by mass spectrometry. Journal of Lipid Research $\mathbf{5 1}$ 1218-1227.

Flood MR \& Wiebold JL 1988 Glucose metabolism by preimplantation pig embryos. Journal of Reproduction and Fertility 84 7-12.

Gandhi AP, Lane M, Gardner DK \& Krisher RL 2001 Substrate utilization in porcine embryos cultured in NCSU23 and G1.2/G2.2 sequential culture media. Molecular Reproduction \& Development 58 269-275.

Gardner DK, Lane M \& Batt P 1993 Uptake and metabolism of pyruvate and glucose by individual sheep preattachment embryos developed in vivo. Molecular Reproduction \& Development 36 313-319.

Gardner DK, Lane M, Stevens J \& Schoolcraft WB 2001 Noninvasive assessment of human embryo nutrient consumption as a measure of developmental potential. Fertility \& Sterility 76 1175-1180.

Gardner DK \& Leese HJ 1987 Assessment of embryo viability prior to transfer by the noninvasive measurement of glucose uptake. Journal of Experimental Zoology 242 103-105.

Gardner DK \& Leese HJ 1990 Concentrations of nutrients in mouse oviduct fluid and their effects on embryo development and metabolism in vitro. Journal of Reproduction \& Fertility 88 361-368.

Gonzalez-Fernandez R, Gaytan F, Martinez-Galisteo E, Porras P, Padilla CA, Sanchez Criado JE \& Barcena JA 2005 Expression of glutaredoxin (thioltransferase) in the rat ovary during the oestrous cycle and postnatal development. Journal of Molecular Endocrinology 34 625-635.

Hardarson T, Ahlstrom A, Rogberg L, Botros L, Hillensjo T, Westlander G, Sakkas D \& Wikland M 2012 Non-invasive metabolomic profiling of Day 2 and 5 embryo culture medium: a prospective randomized trial. Human Reproduction 27 89-96.

Harvey AJ, Kind KL \& Thompson JG 2002 REDOX regulation of early embryonic development. Reproduction 123 479-486.

Harvey AJ, Kind KL \& Thompson JG 2004 Effect of the oxidative phosphorylation uncoupler 2,4-dinitrophenol on hypoxia-inducible factor-regulated gene expression in bovine blastocysts. Reproduction, Fertility, \& Development 16 665-673.

Hashimoto S, Minami N, Takakura R, Yamada M, Imai H \& Kashima N 2000 Low oxygen tension during in vitro 
maturation is beneficial for supporting the subsequent development of bovine cumulus oocyte complexes. Molecular Reproduction and Development 57 353360.

Heller DT, Cahill DM \& Schultz RM 1981 Biochemical studies of mammalian oogenesis: metabolic cooperativity between granulosa cells and growing mouse oocytes. Developmental Biology 84 455-464.

Hemmings KE, Leese HJ \& Picton HM 2012 Amino acid turnover by bovine oocytes provides an index of oocyte developmental competence in vitro. Biology of Reproduction 86 165, 161-112.

Herrick JR, Brad AM \& Krisher RL 2006 Chemical manipulation of glucose metabolism in porcine oocytes: effects on nuclear and cytoplasmic maturation in vitro. Reproduction 131 289-298.

Herrick JR, Brad AM, Krisher RL \& Pope WF 2003 Relationship between intracellular adenosine triphosphate or glutathione concentration and in vivo developmental potential of porcine oocytes. Animal Reproduction Science 78 123-131.

Hewitson LC, Martin KL \& Leese HJ 1996 Effects of metabolic inhibitors on mouse preimplantation embryo development and the energy metabolism of isolated inner cell masses. Molecular Reproduction and Development 43 323-330.

Houghton FD, Hawkhead JA, Humpherson PG, Hogg JE, Balen AH, Rutherford AJ \& Leese HJ 2002 Noninvasive amino acid turnover predicts human embryo developmental capacity. Human Reproduction $\mathbf{1 7}$ 999-1005.

Hugentobler SA, Humpherson PG, Leese HJ, Sreenan JM \& Morris DG 2008 Energy substrates in bovine oviduct and uterine fluid and blood plasma during the oestrous cycle. Molecular Reproduction \& Development 75 496-503.

Hugentobler SA, Sreenan JM, Humpherson PG, Leese HJ, Diskin MG \& Morris DG 2010 Effects of changes in the concentration of systemic progesterone on ions, amino acids and energy substrates in cattle oviduct and uterine fluid and blood. Reproduction, Fertility, \& Development 22 684-694.

Kikuchi K, Ekwall H, Tienthai P, Kawai Y, Noguchi J, Kaneko H \& Rodriguez-Martinez H 2002 Morphological features of lipid droplet transition during porcine oocyte fertilisation and early embryonic development to blastocyst in vivo and in vitro. Zygote 10 355-366.

Kikuchi K, Kashiwazaki N, Noguchi J, Shimada A, Takahashi R, Hirabayashi M, Shino M, Ueda M \& Kaneko H 1999 Developmental competence, after transfer to recipients, of porcine oocytes matured, fertilized and cultured in vitro. Biology of Reproduction 60 336-340.

Kim K, Park S \& Roh S 2012 Lipid-rich blastomeres in the two-cell stage of porcine parthenotes show bias toward contributing to the embryonic part. Anim Reprod Sci 130 91-98.

Krisher R 1999 Exposure of bovine oocytes to perturbants of the pentose phosphate pathway affects subsequent embryonic development. Biology of Reproduction 60 (Suppl. 1) 390.

Krisher RL \& Bavister BD 1999 Enhanced glycolysis after maturation of bovine oocytes in vitro is associated with increased developmental competence. Molecular Reproduction \& Development 53 19-26.

Krisher RL, Brad AM, Herrick JR, Sparman ML \& Swain JE 2007 A comparative analysis of metabolism and viability in porcine oocytes during in vitro maturation. Animal Reproduction Science 98 72-96.

Krisher RL, Lane M \& Bavister BD 1999 Developmental competence and metabolism of bovine embryos cultured in semi-defined and defined culture media. Biology of Reproduction 60 1345-1352.

Krisher RL \& Prather RS 2012 A Role for the Warburg Effect in Preimplantation Embryo Development:Metabolic Modification to Support Rapid Cell Proliferation. Molecular Reproduction \& Development 79 311-320.

Kumar A \& Bachhawat AK 2012 Pyroglutamic acid: throwing light in a lightly studies metabolite. Current Science 102 288-297.

Lane M \& Gardner DK 1998 Amino acids and vitamins prevent culture-induced metabolic perturbations and associated loss of viability of mouse blastocysts. Human Reproduction 13 991-997.

Lane M \& Gardner DK 2005 Mitochondrial malateaspartate shuttle regulates mouse embryo nutrient consumption. Journal of Biological Chemistry $\mathbf{2 8 0}$ 18361-18367.

Lane M \& Gardner DK 2007 Embryo culture medium: which is the best? Best Practice \& Research in Clinical Obstetrics \& Gynaecology 21 83-100.

Leese HJ 2002 Quiet please, do not disturb: a hypothesis of embryo metabolism and viability. Bioessays 24 845-849.

Leese HJ \& Barton AM 1984 Pyruvate and glucose uptake by mouse ova and preimplantation embryos. Journal of Reproduction and Fertility 72 9-13.

Leese HJ, Baumann CG, Brison DR, McEvoy TG \& Sturmey RG 2008 Metabolism of the viable mammalian embryo: quietness revisited. Molecular Human Reproduction 14 667-672.

Leese HJ, Sturmey RG, Baumann CG \& McEvoy TG 2007 Embryo viability and metabolism: obeying the quiet rules. Human Reproduction 22 3047-3050.

Leroy JLMR, Vanholder T, Mateusen B, Christophe A, Opsomer G, de Kruif A, Genicot G \& Van Soom A 2005 Non-esterified fatty acids in follicular fluid of dairy cows and their effect on developmental capacity of bovine oocytes in vitro. Reproduction 130 485-495.

Li R, Whitworth K, Lai L, Wax D, Spate L, Murphy CN, Rieke A, Isom C, Hao Y, Zhong Z, Katayama M, Schatten H \& Prather RS 2007 Concentration and composition of free amino acids and osmolalities of porcine oviductal and uterine fluid and their effects on development of porcine IVF embryos. Molecular Reproduction and Development 74 1228-1235.

Machaty Z, Day BN \& Prather RS 1998 Development of early porcine embryos in vitro and in vivo. Biology of Reproduction 59 451-455. 
Machaty Z, Thompson JG, Abeydeera LR, Day BN \& Prather RS 2001 Inhibitors of mitochondrial ATP production at the time of compaction improve development of in vitro produced porcine embryos. Molecular Reproduction and Development 58 39-44.

Marei WF, Wathes DC \& Fouladi-Nashta AA 2010 Impact of linoleic acid on bovine oocyte maturation and embryo development. Reproduction 139 979-988.

Marhuenda-Egea F, Gonsálvez-Álvarez R, MartínezSabater E, Lledó B, Ten J \& Bernabeu R 2011 Improving human embryos selection in IVF: noninvasive metabolomic and chemometric approach. Metabolomics 7 247-256.

Mito T, Yoshioka K, Yamashita S, Suzuki C, Noguchi M \& Hoshi H 2012 Glucose and glycine synergistically enhance the in vitro development of porcine blastocysts in a chemically defined medium. Reproduction, Fertility and Development 24 443-450.

Montani DA, Cordeiro FB, Regiani T, Victorino AB, Pilau EJ, Gozzo FC, Ferreira CR, Fraietta R \& Lo Turco EG 2012 The follicular microenviroment as a predictor of pregnancy: MALDI-TOF MS lipid profile in cumulus cells. Journal of Assisted Reproduction and Genetics 29 1289-1297.

Nagy ZP, Sakkas D \& Behr B 2008 Symposium: innovative techniques in human embryo viability assessment. Non-invasive assessment of embryo viability by metabolomic profiling of culture media ('metabolomics'). Reprod Biomed Online 17 502-507.

Nichol R, Hunter RH, Gardner DK, Leese HJ \& Cooke GM 1992 Concentrations of energy substrates in oviductal fluid and blood plasma of pigs during the peri-ovulatory period. Journal of Reproduction and Fertility 96 699-707.

O'Brien JK, Dwarte D, Ryan JP, Maxwell WMC \& Evans G 1996 Developmental capacity, energy metabolism and ultrastructure of mature oocytes from prepubertal and adult sheep. Reproduction, Fertility and Development 8 1029-1037.

Orsi NM \& Leese HJ 2004 Ammonium exposure and pyruvate affect the amino acid metabolism of bovine blastocysts in vitro. Reproduction 127 131-140.

Ozawa M, Nagai T, Fahrudin M, Karja NWK, Kaneko H, Noguchi J, Ohnuma K \& Kikuchi K 2006 Addition of glutathione or thioredoxin to culture medium reduces intracellular redox status of porcine IVM/IVF embryos, resulting in improved development to the blastocyst stage. Molecular Reproduction \& Development 73 998-1007.

Partridge RJ \& Leese HJ 1996 Consumption of amino acids by bovine preimplantation embryos. Reproduction Fertility and Development 8 945-950.

Petters RM, Johnson BH, Reed ML \& Archibong AE 1990 Glucose, glutamine and inorganic phosphate in early development of the pig embryo in vitro. Journal of Reproduction and Fertility 89 269-275.

Petters RM \& Wells KD 1993 Culture of pig embryos. Journal of Reproduction and Fertility-Supplement 48 61-73.
Picton HM, Elder K, Houghton FD, Hawkhead JA, Rutherford AJ, Hogg JE, Leese HJ \& Harris SE 2010 Association between amino acid turnover and chromosome aneuploidy during human preimplantation embryo development in vitro. Molecular Human Reproduction 16 557-569.

Quinn P \& Wales RG 1973 The relationships between the ATP content of preimplantation mouse embryos and their development in vitro during culture. Journal of Reproduction and Fertility 35 301-309.

Redel BK, Brown AN, Spate LD, Whitworth KM, Green JA \& Prather RS 2012 Glycolysis in preimplantation development is partially controlled by the Warburg Effect. Molecular Reproduction \& Development 79 262-271.

Rieger D \& Loskutoff NM 1994 Changes in the metabolism of glucose, pyruvate, glutamine and glycine during maturation of cattle oocytes in vitro. Journal of Reproduction and Fertility 100 257-262.

Rieger D, Loskutoff NM \& Betteridge KJ 1992 Developmentally related changes in the uptake and metabolism of glucose, glutamine and pyruvate by cattle embryos produced in vitro. Reproduction, Fertility and Development 4 547-557.

Rieger D, McGowan LT, Cox SF, Pugh PA \& Thompson JG 2002 Effect of 2,4-dinitrophenol on the energy metabolism of cattle embryos produced by in vitro fertilization and culture. Reproduction, Fertility, \& Development 14 339-343.

Romek M, Gajda B, Krzysztofowicz E, Kepczynski M \& Smorag Z 2011 New technique to quantify the lipid composition of lipid droplets in porcine oocytes and pre-implantation embryos using Nile Red fluorescent probe. Theriogenology 75 42-54.

Romek M, Gajda B, Krzysztofowicz E \& Smorag Z 2010 Changes of lipid composition in non-cultured and cultured porcine embryos. Theriogenology $\mathbf{7 4}$ 265-276.

Rosenkrans CF, Jr. \& First NL 1994 Effect of free amino acids and vitamins on cleavage and developmental rate of bovine zygotes in vitro. Journal of Animal Sciences 72 434-437.

Saito T, Hiroi M \& Kato T 1994 Development of glucose utilization studied in single oocytes and preimplantation embryos from mice. Biology of Reproduction 50 266-270.

Sakkas D, Morita H, Yamashita N, Kato O, Botros L, Roos P \& Seli E 2008 Evaluation of Embryo Quality by Metabolomics: A New Strategy to Aid Single Embryo Transfer. J Mamm Ova Res 25 26-31.

Scott R, Seli E, Miller K, Sakkas D, Scott K \& Burns DH 2008 Noninvasive metabolomic profiling of human embryo culture media using Raman spectroscopy predicts embryonic reproductive potential: a prospective blinded pilot study. Fertility and Sterility 90 77-83.

Singh B, Meng L, Rutledge JM \& Armstrong DT 1997 Effects of epidermal growth factor and follicle stimulating hormone during in vitro maturation 
on cytoplasmic maturation of porcine oocytes. . Molecular Reproduction and Development 46 401407.

Somfai T, Kaneda M, Akagi S, Watanabe S, Haraguchi S, Mizutani E, Dang-Nguyen TQ, Geshi M, Kikuchi K \& Nagai T 2011a Enhancement of lipid metabolism with L-carnitine during in vitro maturation improves nuclear maturation and cleavage ability of follicular porcine oocytes. Reproduction, Fertility, \& Development 23 912-920.

Somfai T, Kaneda M, Akagi S, Watanabe S, Haraguchi S, Mizutani E, Dang-Nguyen TQ, Geshi M, Kikuchi K \& Nagai T 2011b Enhancement of lipid metabolism with $\mathrm{L}$-carnitine during in vitro maturation improves nuclear maturation and cleavage ability of follicular porcine oocytes. Reproduction, Fertility and Development 23 912-920.

Spindler RE, Pukazhenthi BS \& Wildt DE 2000 Oocyte metabolism predicts the development of cat embryos to blastocyst in vitro. Molecular Reproduction and Development 56 163-171.

Steeves TE \& Gardner DK 1999 Metabolism of glucose, pyruvate, and glutamine during the maturation of oocytes derived from pre-pubertal and adult cows. Molecular Reproduction \& Development 54 92-101.

Sturmey RG, Bermejo-Alvarez P, Gutierrez-Adan A, Rizos D, Leese HJ \& Lonergan P 2010 Amino acid metabolism of bovine blastocysts: a biomarker of sex and viability.[Erratum appears in Mol Reprod Dev. 2010 May;77(5):472]. Molecular Reproduction \& Development 77 285-296.

Sturmey RG, Brison DR \& Leese HJ 2008 Symposium: innovative techniques in human embryo viability assessment. Assessing embryo viability by measurement of amino acid turnover. Reproductive BioMedicine Online 17 486-496.

Sturmey RG, Hawkhead JA, Barker EA \& Leese HJ 2009a DNA damage and metabolic activity in the preimplantation embryo. Human Reproduction 24 81-91.

Sturmey RG \& Leese HJ 2003a Energy metabolism in pig oocytes and early embryos. Reproduction 126 197-204.

Sturmey RG \& Leese HJ 2003b Energy metabolism in pig oocytes and early embryos. Reproduction 126 197-204.

Sturmey RG \& Leese HJ 2008 Role of glucose and fatty acid metabolism in porcine early embryo development. Reproduction, Fertility and Development 20149.

Sturmey RG, O'Toole PJ \& Leese HJ 2006 Fluorescence resonance energy transfer analysis of mitochondrial:lipid association in the porcine oocyte. Reproduction 132 829-837.

Sturmey RG, Reis A, Leese HJ \& McEvoy TG 2009b Role of fatty acids in energy provision during oocyte maturation and early embryo development. Reproduction in Domestic Animals 44 Suppl 3 50-58.

Sudano MJ, Santos VG, Tata A, Ferreira CR, Paschoal DM, Machado R, Buratini J, Eberlin MN \& Landim-
Alvarenga FD 2012 Phosphatidylcholine and Sphingomyelin Profiles Vary in Bos taurus indicus and Bos taurus taurus In Vitro- and In Vivo-Produced Blastocysts. Biology of Reproduction 87130.

Sutton-McDowall ML, Gilchrist RB \& Thompson JG 2004 Cumulus expansion and glucose utilisation by bovine cumulus-oocyte complexes during in vitro maturation: the influence of glucosamine and follicle-stimulating hormone. Reproduction 128 313-319.

Sutton-McDowall ML, Gilchrist RB \& Thompson JG 2010 The pivotal role of glucose metabolism in determining oocyte developmental competence. Reproduction 139 685-695.

Sutton ML, Gilchrist RB \& Thompson JG 2003 Effects of in-vivo and in-vitro environments on the metabolism of the cumulus-oocyte complex and its influence on oocyte developmental capacity. Human Reproduction Update 9 35-48.

Swain JE, Bormann CL, Clark SG, Walters EM, Wheeler MB \& Krisher RL 2002 Use of energy substrates by various stage preimplantation pig embryos produced in vivo and in vitro. Reproduction 123 253-260.

Takahashi Y \& First NL 1992 In vitro development of bovine one cell embryos: influence of glucose, lactate, pyruvate, amino acids and vitamins. Theriogenology 37 963-978.

Thompson JG, McNaughton C, Gasparrini B, McGowan LT \& Tervit HR 2000 Effect of inhibitors and uncouplers of oxidative phosphorylation during compaction and blastulation of bovine embryos cultured in vitro. Journal of Reproduction \& Fertility 118 47-55.

Thompson JG, Simpson AC, Pugh PA, Wright RW, Jr. \& Tervit HR 1991 Glucose utilization by sheep embryos derived in vivo and in vitro. Reproduction, Fertility, \& Development 3 571-576.

Tsutsumi O, Satoh K, Taketani Y \& Kato T 1992 Determination of enzyme activities of energy metabolism in the maturing rat oocyte. Molecular Reproduction and Development. 33 333-337.

Van Blerkom J, Davis PW \& Lee J 1995 ATP content of human oocytes and developmental potential and outcome after in vitro fertilization and embryo transfer. Human Reproduction 10 415-424.

Van Hoeck V, Sturmey RG, Bermejo-Alvarez P, Rizos D, Gutierrez-Adan A, Leese HJ, Bols PEJ \& Leroy J 2011 Elevated non-esterified fatty acid concentrations during bovine oocyte maturation compromise early embryo physiology. PLOS ONE [Electronic Resource] 6 e23183.

Vergouw CG, Kieslinger DC, Kostelijk EH, Botros LL, Schats R, Hompes PG, Sakkas D \& Lambalk CB 2012 Day 3 embryo selection by metabolomic profiling of culture medium with near-infrared spectroscopy as an adjunct to morphology: a randomized controlled trial. Human Reproduction 27 2304-2311.

Wale PL \& Gardner DK 2012 Oxygen regulates amino acid turnover and carbohydrate uptake during the preimplantation period of mouse embryo development. Biology of Reproduction 87 24, 21-28. 
Wu GQ, Jia BY, Li JJ, Fu XW, Zhou GB, Hou YP \& Zhu SE 2011 L-carnitine enhances oocyte maturation and development of parthenogenetic embryos in pigs. Theriogenology 76 785-793.

Yoshida M, Mizoguchi Y, Ishigaki K, Kojima T \& Nagai T 1993 Birth of piglets derived from in vitro fertilization of pig oocytes matured in vitro. Theriogenology 39 1303-1311.
Yuan Y, Wheeler MB \& Krisher RL 2012 Disrupted redox homeostasis and aberrant redox gene expression in porcine oocytes contribute to decreased developmental competence. Biology of Reproduction 8778.

Zuelke KA \& Brackett BG 1992 Effects of luteinizing hormone on glucose metabolism in cumulus-enclosed bovine oocytes matured in vitro. Endocrinology 131 2690-2696. 